\title{
GENERAL PROJECTION SYSTEMS AND RELAXED COCOERCIVE NONLINEAR VARIATIONAL INEQUALITIES
}

\author{
RAM U. VERMA ${ }^{1}$
}

(Received 8 April, 2007)

\begin{abstract}
We explore the solvability of a general system of nonlinear relaxed cocoercive variational inequality (SNVI) problems based on a new projection system for the direct product of two nonempty closed and convex subsets of real Hilbert spaces.

2000 Mathematics subject classification: primary 49J40, 65B05; secondary 47H20.

Keywords and phrases: relaxed cocoercive mappings, approximation solvability, projection system, system of nonlinear relaxed cocoercive variational inequalities.
\end{abstract}

\section{Introduction}

Projection-type systems are frequently used in convergence analysis for solutions of variational inequality problems arising in several fields, for instance, in complementarity theory, convex quadratic programming, optimization and control theory, and variational problems. Projection methods (sometimes referred to as Galerkin methods) are also applied in different contexts, especially to inner approximation schemes for $A$-proper nonlinear equations [9] where solutions are approximated as strong limits of solutions of corresponding simpler systems of finite-dimensional equations. Recently, Chang et al. [1] considered the application of the general two-step model [7] for projection methods to the approximation solvability of nonlinear strongly monotone inequality problems in Hilbert spaces. They generalized the iterative algorithm used in $[6,7]$ along with some special iterative algorithms of interest. In this paper we explore, based on a general system of projection-type methods, the approximation solvability of a system of nonlinear relaxed cocoercive variational inequalities in Hilbert spaces. The notion of relaxed cocoercivity generalizes the notion of monotonicity as well as

\footnotetext{
'Department of Mathematics, University of Toledo, Toledo, Ohio 43606, USA; email: verma99@msn.com.

(C) Australian Mathematical Society 2007, Serial-fee code 1446-1811/07
} 
of strong monotonicity. The obtained results extend and improve the results in [1], [5], [6] and [7]. For more details, we refer the reader to [1-9].

Let $H_{1}$ and $H_{2}$ be two real Hilbert spaces with inner product $(\cdot, \cdot)$ and norm $\|\cdot\|$. Let $S: K_{1} \times K_{2} \rightarrow H_{1}$ and $T: K_{1} \times K_{2} \rightarrow H_{2}$ be any mappings on $K_{1} \times K_{2}$, where $K_{1}$ and $K_{2}$ are nonempty closed convex subsets of $H_{1}$ and $H_{2}$, respectively. We consider a system of nonlinear variational inequality (SNVI) problems: find an element $\left(x^{*}, y^{*}\right) \in K_{1} \times K_{2}$ such that

$$
\begin{array}{ll}
\left\langle\rho S\left(x^{*}, y^{*}\right), x-x^{*}\right\rangle \geq 0 & \forall x \in K_{1} \quad \text { and } \\
\left\langle\eta T\left(x^{*}, y^{*}\right), y-y^{*}\right\rangle \geq 0 & \forall y \in K_{2},
\end{array}
$$

where $\rho, \eta>0$.

The SNVI (1.1)-(1.2) problem is equivalent to the projection formulae

$$
\begin{array}{ll}
x^{*}=P_{K}\left[x^{*}-\rho S\left(x^{*}, y^{*}\right)\right] & \text { for } \rho>0 \text { and } \\
y^{*}=Q_{K}\left[y^{*}-\eta T\left(x^{*}, y^{*}\right)\right] & \text { for } \eta>0,
\end{array}
$$

where $P_{K}$ is the projection of $H_{1}$ onto $K_{1}$ and $Q_{K}$ is the projection of $H_{2}$ onto $K_{2}$.

We note that the SNVI (1.1)-(1.2) problem extends the NVI problem: determine an element $x^{*} \in K_{1}$ such that

$$
\left\langle S\left(x^{*}\right), x-x^{*}\right\rangle \geq 0, \quad \forall x \in K_{1} .
$$

Also, we note that the SNVI (1.1)-(1.2) problem is equivalent to a system of nonlinear complementarities (SNCs): find $\left(x^{*}, y^{*}\right) \in K_{1} \times K_{2}$ such that $S\left(x^{*}, y^{*}\right) \in K_{1}^{*}$, $T\left(x^{*}, y^{*}\right) \in K_{2}^{*}$, and

$$
\left\langle\rho S\left(x^{*}, y^{*}\right), x^{*}\right\rangle=0 \quad \text { for } \rho>0, \quad\left\langle\eta T\left(x^{*}, y^{*}\right), y^{*}\right\rangle=0 \quad \text { for } \eta>0,
$$

where $K_{1}^{*}$ and $K_{2}^{*}$, respectively, are polar cones to $K_{1}$ and $K_{2}$ defined by

$$
\begin{aligned}
& K_{1}^{*}=\left\{f \in H_{1}:\langle f, x\rangle \geq 0, \quad \forall x \in K_{1}\right\} \quad \text { and } \\
& K_{2}^{*}=\left\{g \in H_{2}:\{g, y\rangle \geq 0, \quad \forall g \in K_{2}\right\} .
\end{aligned}
$$

Now, we recall some auxiliary results and notions crucial to the problem at hand.

LEMMA 1.1. For an element $z \in H$, we have $x \in K$ and $\langle x-z, y-x\rangle \geq 0$, $\forall y \in K$ if and only if $x=P_{K}(z)$.

LEMMA 1.2 ([2]). Let $\left\{\alpha^{k}\right\},\left\{\beta^{k}\right\}$ and $\left\{\gamma^{k}\right\}$ be three nonnegative sequences such that

$$
\alpha^{k+1} \leq\left(1-t^{k}\right) \alpha^{k}+\beta^{k}+\gamma^{k} \quad \text { for } k=0,1,2, \ldots,
$$

where $t^{k} \in[0,1], \sum_{k=0}^{\infty} t^{k}=\infty, \beta^{k}=o\left(t^{k}\right)$ and $\sum_{k=0}^{\infty} \gamma^{k}<\infty$. Then $\alpha^{k} \rightarrow 0$ as $k \rightarrow \infty$. 
For $H$ a Hilbert space, a mapping $T: H \rightarrow H$ is called monotone if $\forall x, y \in H$, $\langle T(x)-T(y), x-y\rangle \geq 0$. The mapping $T$ is $(r)$-strongly monotone if for each $x, y \in H$, we have

$$
\langle T(x)-T(y), x-y\rangle \geq r\|x-y\|^{2} \text { for a constant } r>0 .
$$

This implies that $\|T(x)-T(y)\| \geq r\|x-y\|$, that is, $T$ is ( $r$ )-expansive, and when $r=1$, it is expansive. The mapping $T$ is called (s)-Lipschitz continuous (or Lipschitzian) if there exists a constant $s \geq 0$ such that $\|T(x)-T(y)\| \leq s\|x-y\|$, $\forall x, y \in H$. The mapping $T$ is called $(\mu)$-cocoercive if for each $x, y \in H$, we have

$$
\langle T(x)-T(y), x-y\rangle \geq \mu\|T(x)-T(y)\|^{2} \text { for a constant } \mu>0 .
$$

Clearly, every $(\mu)$-cocoercive mapping $T$ is $(1 / \mu)$-Lipschitz continuous. We note that $T$ is called relaxed $(\gamma)$-cocoercive if there exists a constant $\gamma>0$ such that

$$
\langle T(x)-T(y), x-y\rangle \geq(-\gamma)\|T(x)-T(y)\|^{2}, \quad \forall x, y \in H .
$$

We say that $T$ is $(r)$-strongly pseudomonotone if there exists a constant $r>0$ such that

$$
\langle T(y), x-y\rangle \geq 0 \quad \Rightarrow \quad\langle T(x), x-y\rangle \geq r\|x-y\|^{2}, \quad \forall x, y \in H,
$$

and that $T$ is relaxed $(\gamma, r)$-cocoercive if there exist constants $\gamma, r>0$ such that

$$
\langle T(x)-T(y), x-y\rangle \geq(-\gamma)\|T(x)-T(y)\|^{2}+r\|x-y\|^{2} .
$$

This implies

$$
\langle T(x)-T(y), x-y\rangle \geq(-\gamma)\|T(x)-T(y)\|^{2},
$$

that is, $T$ is relaxed $(\gamma)$-cocoercive.

We define $\mathrm{T}$ to be relaxed $(\gamma, r)$-pseudococoercive if there exist positive constants $\gamma$ and $r$ such that $\forall x, y \in H$

$$
\langle T(y), x-y\rangle \geq 0 \quad \Rightarrow \quad\langle T(x), x-y\rangle \geq(-\gamma)\|T(x)-T(y)\|^{2}+r\|x-y\|^{2} .
$$

\section{General projection methods}

In this section, we discuss the approximation-solvability of the SNVI (1.1)-(1.2) problem based on the following algorithms.

ALGORITHM 1. For an arbitrarily chosen initial point $\left(x^{0}, y^{0}\right) \in K_{1} \times K_{2}$, compute the sequences $\left\{x^{k}\right\}$ and $\left\{y^{k}\right\}$ such that

$$
\begin{aligned}
x^{k+1} & =\left(1-a^{k}-b^{k}\right) x^{k}+a^{k} P_{K}\left[x^{k}-\rho S\left(x^{k}, y^{k}\right)\right]+b^{k} u^{k} \text { and } \\
y^{k+1} & =\left(1-\alpha^{k}-\beta^{k}\right) y^{k}+\alpha^{k} Q_{k}\left[y^{k}-\eta T\left(x^{k}, y^{k}\right)\right]+\beta^{k} v^{k},
\end{aligned}
$$


where $\rho, \eta>0$ are constants, and $\left\{u^{k}\right\}$ and $\left\{v^{k}\right\}$, respectively, are bounded sequences in $K_{1}$ and $K_{2}$. The sequences $\left\{a^{k}\right\},\left\{b^{k}\right\},\left\{\alpha^{k}\right\}$ and $\left\{\beta^{k}\right\}$ are in $[0,1]$ with $(k \geq 0)$

$$
0 \leq a^{k}+b^{k} \leq 1, \quad 0 \leq \alpha^{k}+\beta^{k} \leq 1
$$

ALGORITHM 2. For an arbitrarily chosen initial point $\left(x^{0}, y^{0}\right) \in K_{1} \times K_{2}$, compute the sequences $\left\{x^{k}\right\}$ and $\left\{y^{k}\right\}$ such that

$$
\begin{aligned}
x^{k+1} & =\left(1-a^{k}-b^{k}\right) x^{k}+a^{k} P_{K}\left[x^{k}-\rho S\left(x^{k}, y^{k}\right)\right]+b^{k} u^{k} \text { and } \\
y^{k+1} & =\left(1-a^{k}-b^{k}\right) y^{k}+a^{k} Q_{K}\left[y^{k}-\eta T\left(x^{k}, y^{k}\right)\right]+b^{k} v^{k},
\end{aligned}
$$

where $\rho, \eta>0$ are constants, and $\left\{u^{k}\right\}$ and $\left\{v^{k}\right\}$, respectively, are bounded sequences in $K_{1}$ and $K_{2}$. The sequences $\left\{a^{k}\right\}$ and $\left\{b^{k}\right\}$ are in $[0,1]$ with $(k \geq 0)$

$$
0 \leq a^{k}+b^{k} \leq 1
$$

ALGORITHM 3. For an arbitrarily chosen initial point $\left(x^{0}, y^{0}\right) \in K_{1} \times K_{2}$, compute the sequences $\left\{x^{k}\right\}$ and $\left\{y^{k}\right\}$ such that

$$
\begin{aligned}
x^{k+1} & =\left(1-a^{k}\right) x^{k}+a^{k} P_{K}\left[x^{k}-\rho S\left(x^{k}, y^{k}\right)\right] \text { and } \\
y^{k+1} & =\left(1-a^{k}\right) y^{k}+a^{k} Q_{K}\left[y^{k}-\eta T\left(x^{k}, y^{k}\right)\right],
\end{aligned}
$$

where $\rho, \eta>0$ are constants. The sequence $\left\{a^{k}\right\} \subset[0,1]$ for $k \geq 0$.

Next, we consider, based on Algorithm 2, the approximation solvability of the SNVI (1.1)-(1.2) problem involving strongly monotone and Lipschitz continuous mappings in Hilbert space settings.

THEOREM 2.1. Let $H_{1}$ and $H_{2}$ be two real Hilbert spaces and $K_{1}$ and $K_{2}$, respectively, be nonempty closed convex subsets of $H_{1}$ and $H_{2}$. Let $S: K_{1} \times K_{2} \rightarrow H_{1}$ be relaxed $(\gamma, r)$-cocoercive and $(\mu)$-Lipschitz continuous in the first variable and let $S$ be ( $\nu)$-Lipschitz continuous in the second variable. Let $T: K_{1} \times K_{2} \rightarrow H_{2}$ be relaxed $(\lambda, s)$-cocoercive and $(\beta)$-Lipschitz continuous in the second variable and let $T$ be $(\tau)$-Lipschitz continuous in the first variable. Let $\|\cdot\|^{*}$ denote the norm on $\mathrm{H}_{1} \times \mathrm{H}_{2}$ defined by

$$
\|(x, y)\|^{*}=(\|x\|+\|y\|) \quad \forall(x, y) \in H_{1} \times H_{2} .
$$

In addition, let

$$
\begin{aligned}
\theta+n \tau & =\sqrt{1-2 \rho r+2 \rho \gamma \mu^{2}+\rho^{2} \mu^{2}}+\eta \tau<1, \\
\sigma+\rho \nu & =\sqrt{1-2 \eta r+2 \eta \lambda \beta^{2}+\eta^{2} \beta^{2}}+\rho \nu<1
\end{aligned}
$$

let $\left(x^{*}, y^{*}\right) \in K_{1} \times K_{2}$ form a solution to the SNVI (1.1)-(1.2) problem, and let sequences $\left\{x^{k}\right\}$ and $\left\{y^{k}\right\}$ be generated by Algorithm 2 . 
Furthermore, let

(i) $0 \leq a^{k}+b^{k} \leq 1$,

(ii) $\sum_{k=0}^{\infty} a^{k}=\infty$ and $\sum_{k=0}^{\infty} b^{k}<\infty$, and

(iii) $0<\rho<2 r / \mu^{2}$ and $0<\eta<2 s / \beta^{2}$.

Then the sequence $\left\{\left(x^{k}, y^{k}\right)\right\}$ converges to $\left(x^{*}, y^{*}\right)$.

PROOF. Since $\left(x^{*}, y^{*}\right) \in K_{1} \times K_{2}$ forms a solution to the SNVI(1.1)-(1.2) problem, it follows that

$$
x^{*}=P_{K}\left[x^{*}-\rho S\left(x^{*}, y^{*}\right)\right] \text { and } y^{*}=Q_{K}\left[y^{*}-\eta T\left(x^{*}, y^{*}\right)\right] .
$$

Applying Algorithm 2, we have

$$
\begin{aligned}
\| x^{k+1}- & x^{*} \| \\
= & \|\left(1-a^{k}-b^{k}\right) x^{k}+a^{k} P_{K}\left[x^{k}-\rho S\left(x^{k}, y^{k}\right)\right]+b^{k} u^{k} \\
& -\left(1-a^{k}-b^{k}\right) x^{*}-a^{k} P_{K}\left[x^{*}-\rho S\left(x^{*}, y^{*}\right)\right]-b^{k} x^{*} \| \\
\leq & \left(1-a^{k}-b^{k}\right)\left\|x^{k}-x^{*}\right\| \\
& +a^{k}\left\|P_{K}\left[x^{k}-\rho S\left(x^{k}, y^{k}\right)\right]-P_{K}\left[x^{*}-\rho S\left(x^{*}, y^{*}\right)\right]\right\|+M b^{k} \\
\leq & \left(1-a^{k}\right)\left\|x^{k}-x^{*}\right\| \\
& +a^{k}\left\|x^{k}-x^{*}-\rho\left[S\left(x^{k}, y^{k}\right)-S\left(x^{*}, y^{k}\right)+S\left(x^{*}, y^{k}\right)-S\left(x^{*}, y^{*}\right)\right]\right\|+M b^{k} \\
\leq & \left(1-a^{k}\right)\left\|x^{k}-x^{*}\right\|+a^{k}\left\|x^{k}-x^{*}-\rho\left[S\left(x^{k}, y^{k}\right)-S\left(x^{*}, y^{k}\right)\right]\right\| \\
& +a^{k} \rho\left\|\left[S\left(x^{*}, y^{k}\right)-S\left(x^{*}, y^{*}\right)\right]\right\|+M b^{k},
\end{aligned}
$$

where $M=\max \left\{\sup \left\|u^{k}-x^{*}\right\|, \sup \left\|v^{k}-y^{*}\right\|\right\}<\infty$.

Since $S$ is relaxed $(\gamma, r)$-cocoercive and $(\mu)$-Lipschitz continuous in the first variable, and $S$ is $(\nu)$-Lipschitz continuous in the second variable, we have in light of part (i) of Theorem 2.1 that

$$
\begin{aligned}
\| x^{k}- & x^{*}-\rho\left[S\left(x^{k}, y^{k}\right)-S\left(x^{*}, y^{k}\right)\right] \|^{2} \\
= & \left\|x-x^{*}\right\|^{2}-2 \rho\left\langle S\left(x^{k}, y^{k}\right)-S\left(x^{*}, y^{k}\right), x^{k}-x^{*}\right\rangle \\
& +\rho^{2}\left\|S\left(x^{k}, y^{k}\right)-S\left(x^{*}, y^{k}\right)\right\|^{2} \\
= & \left\|x-x^{*}\right\|^{2}-2 \rho\left\langle S\left(x^{k}, y^{k}\right)-S\left(x^{*}, y^{k}\right), x^{k}-x^{*}\right\rangle \\
& +\left(\rho^{2}+2 \rho \gamma\right)\left\|S\left(x^{k}, y^{k}\right)-S\left(x^{*}, y^{k}\right)\right\|^{2} \\
\leq & \left\|x^{k}-x^{*}\right\|^{2}-2 \rho r\left\|x^{k}-x^{*}\right\|^{2}+\left(\rho^{2}+2 \rho \gamma\right) \mu^{2}\left\|x^{k}-x^{*}\right\|^{2} \\
= & {\left[1-2 \rho r+\rho^{2} \mu^{2}+2 \rho \gamma \mu^{2}\right]\left\|x^{k}-x^{*}\right\|^{2} . }
\end{aligned}
$$

As a result, letting $\theta=\sqrt{1-2 \rho r+2 \rho \gamma \mu^{2}+\rho^{2} \mu^{2}}$ we have

$$
\left\|x^{k+1}-x^{*}\right\| \leq\left(1-a^{k}\right)\left\|x^{k}-x^{*}\right\|+a^{k} \theta\left\|x^{k}-x^{*}\right\|+a^{k} \rho \nu\left\|y^{k}-y^{*}\right\|+M b^{k} \text {. }
$$


Similarly, letting $\sigma=\sqrt{1-2 \eta r+2 \eta \lambda \beta^{2}+\eta^{2} \beta^{2}}$ we have

$$
\left\|y^{k+1}-y^{*}\right\| \leq\left(1-a^{k}\right)\left\|y^{k}-y^{*}\right\|+a^{k} \sigma\left\|y^{k}-y^{*}\right\|+a^{k} \eta \tau\left\|x^{k}-x^{*}\right\|+M b^{k} .
$$

It follows from (2.1) and (2.2) that

$$
\begin{aligned}
\| x^{k+1}- & x^{*}\|+\| y^{k+1}-y^{*} \| \\
\leq & \left(1-a^{k}\right)\left\|x^{k}-x^{*}\right\|+a^{k} \theta\left\|x^{k}-x^{*}\right\|+a^{k} \eta \tau\left\|x^{k}-x^{*}\right\|+M b^{k} \\
& +\left(1-a^{k}\right)\left\|y^{k}-y^{*}\right\|+a^{k} \sigma\left\|y^{k}-y^{*}\right\|+a^{k} \rho \nu\left\|y^{k}-y^{*}\right\|+M b^{k} \\
= & {\left[1-(1-\delta) a^{k}\right]\left(\left\|x^{k}-x^{*}\right\|+\left\|y^{k}-y^{*}\right\|\right)+2 M b^{k}, }
\end{aligned}
$$

where $\delta=\max \{\theta+\eta \tau, \sigma+\rho \nu\}$ and $H_{1} \times H_{2}$ is a Banach space under the norm $\|\cdot\|^{*}$.

If we set

$$
\alpha^{k}=\left\|x^{k}-x^{*}\right\|+\left\|y^{k}-y^{*}\right\|, \quad t^{k}=(1-\delta) a^{k}, \quad \beta^{k}=2 M b^{k}
$$

for $k=0,1,2, \ldots$, in Lemma 1.2, and apply (i) and (ii), we conclude that $\left\|x^{k}-x^{*}\right\|+\left\|y^{k}-y^{*}\right\| \rightarrow 0$ as $k \rightarrow \infty$. Hence $\left\|x^{k+1}-x^{*}\right\|+\left\|y^{k+1}-y^{*}\right\| \rightarrow 0$.

Consequently, the sequence $\left\{\left(x^{k}, y^{k}\right)\right\}$ converges strongly to $\left(x^{*}, y^{*}\right)$, a solution to the SNVI (1.1)-(1.2) problem. This completes the proof.

Note that the proof of the following theorem follows rather directly without using Lemma 1.2 .

THEOREM 2.2. Let $H_{1}$ and $H_{2}$ be two real Hilbert spaces and let $K_{1}$ and $K_{2}$, respectively, be nonempty closed convex subsets of $H_{1}$ and $H_{2}$. Let $S: K_{1} \times K_{2} \rightarrow H_{1}$ be relaxed $(\gamma, r)$-cocoercive and $(\mu)$-Lipschitz continuous in the first variable and let $S$ be $(\nu)$-Lipschitz continuous in the second variable. Let $T: K_{1} \times K_{2} \rightarrow H_{2}$ be relaxed $(\lambda, s)$-cocoercive and $(\beta)$-Lipschitz continuous in the second variable and let $T$ be $(\tau)$-Lipschitz continuous in the first variable. Let $\|\cdot\|^{*}$ denote the norm on $H_{1} \times H_{2}$ defined by

$$
\|(x, y)\|^{*}=(\|x\|+\|y\|) \quad \forall(x, y) \in H_{1} \times H_{2} .
$$

In addition, let

$$
\begin{aligned}
& \theta+\eta \tau=\sqrt{1-2 \rho r+2 \rho \gamma \mu^{2}+\rho^{2} \mu^{2}}+\eta \tau<1, \\
& \sigma+\rho \nu=\sqrt{1-2 \eta r+2 \eta \lambda \beta^{2}+\eta^{2} \beta^{2}}+\rho \nu<1,
\end{aligned}
$$

let $\left(x^{*}, y^{*}\right) \in K_{1} \times K_{2}$ form a solution to the SNVI (1.1)-(1.2) problem, and let sequences $\left\{x^{k}\right\}$ and $\left\{y^{k}\right\}$ be generated by Algorithm 3. Furthermore, let

(i) $0 \leq a^{k} \leq 1$, 
(ii) $\sum_{k=0}^{\infty} a^{k}=\infty$ and

(iii) $0<\rho<2 r / \mu^{2}$ and $0<\eta<2 s / \beta^{2}$.

Then the sequence $\left\{\left(x^{k}, y^{k}\right)\right\}$ converges strongly to $\left(x^{*}, y^{*}\right)$.

Proof. Since $\left(x^{*}, y^{*}\right) \in K_{1} \times K_{2}$ forms a solution to the SNVI(1.1)-(1.2) problem, it follows that

$$
x^{*}=P_{K}\left[x^{*}-\rho S\left(x^{*}, y^{*}\right)\right] \quad \text { and } \quad y^{*}=Q_{K}\left[y^{*}-\eta T\left(x^{*}, y^{*}\right)\right] .
$$

Applying Algorithm 3, we have

$$
\begin{aligned}
\left\|x^{k+1}-x^{*}\right\|= & \|\left(1-a^{k}\right) x^{k}+a^{k} P_{K}\left[x^{k}-\rho S\left(x^{k}, y^{k}\right)\right] \\
& -\left(1-a^{k}\right) x^{*}-a^{k} P_{K}\left[x^{*}-\rho S\left(x^{*}, y^{*}\right)\right] \| \\
\leq & \left(1-a^{k}\right)\left\|x^{k}-x^{*}\right\| \\
& +a^{k}\left\|P_{K}\left[x^{k}-\rho S\left(x^{k}, y^{k}\right)\right]-P_{K}\left[x^{*}-\rho S\left(x^{*}, y^{*}\right)\right]\right\| \\
\leq & \left(1-a^{k}\right)\left\|x^{k}-x^{*}\right\| \\
& +a^{k}\left\|x^{k}-x^{*}-\rho\left[S\left(x^{k}, y^{k}\right)-S\left(x^{*}, y^{k}\right)+S\left(x^{*}, y^{k}\right)-S\left(x^{*}, y^{*}\right)\right]\right\| \\
\leq & \left(1-a^{k}\right)\left\|x^{k}-x^{*}\right\|+a^{k}\left\|x^{k}-x^{*}-\rho\left[S\left(x^{k}, y^{k}\right)-S\left(x^{*}, y^{k}\right)\right]\right\| \\
& +a^{k} \rho\left\|\left[S\left(x^{*}, y^{k}\right)-S\left(x^{*}, y^{*}\right)\right]\right\| .
\end{aligned}
$$

Since $S$ is relaxed $(\gamma, r)$-cocoercive and $(\mu)$-Lipschitz continuous in the first variable, and $S$ is $(\nu)$-Lipschitz continuous in the second variable, we have

$$
\begin{aligned}
\| x^{k}- & x^{*}-\rho\left[S\left(x^{k}, y^{k}\right)-S\left(x^{*}, y^{k}\right)\right] \|^{2} \\
= & \left\|x-x^{*}\right\|^{2}-2 \rho\left\langle S\left(x^{k}, y^{k}\right)-S\left(x^{*}, y^{k}\right), x^{k}-x^{*}\right\rangle \\
& +\rho^{2}\left\|S\left(x^{k}, y^{k}\right)-S\left(x^{*}, y^{k}\right)\right\|^{2} \\
= & \left\|x-x^{*}\right\|^{2}-2 \rho\left(S\left(x^{k}, y^{k}\right)-S\left(x^{*}, y^{k}\right), x^{k}-x^{*}\right\rangle \\
& +\rho^{2}\left\|S\left(x^{k}, y^{k}\right)-S\left(x^{*}, y^{k}\right)\right\|^{2} \\
\leq & \left\|x^{k}-x^{*}\right\|^{2}-2 \rho r\left\|x^{k}-x^{*}\right\|^{2}+\rho^{2} \mu^{2}\left\|x^{k}-x^{*}\right\|^{2} \\
& +2 \rho \gamma\left\|S\left(x^{k}, y^{k}\right)-S\left(x^{*}, y^{k}\right)\right\|^{2} \\
\leq & \left\|x^{k}-x^{*}\right\|^{2}-2 \rho r\left\|x^{k}-x^{*}\right\|^{2}+\rho^{2} \mu^{2}\left\|x^{k}-x^{*}\right\|^{2}+2 \rho \gamma \mu^{2}\left\|x^{k}-x^{*}\right\|^{2} \\
= & {\left[1-2 \rho r+\rho^{2} \mu^{2}+2 \rho \gamma \mu^{2}\right]\left\|x^{k}-x^{*}\right\|^{2} . }
\end{aligned}
$$

Setting $\theta=\sqrt{1-2 \rho r+2 \rho \gamma \mu^{2}+\rho^{2} \mu^{2}}$ it follows that

$$
\left\|x^{k+1}-x^{*}\right\| \leq\left(1-a^{k}\right)\left\|x^{k}-x^{*}\right\|+a^{k} \theta\left\|x^{k}-x^{*}\right\|+a^{k} \rho \nu\left\|y^{k}-y^{*}\right\| .
$$

Similarly, setting $\sigma=\sqrt{1-2 \eta r+2 \eta \lambda \beta^{2}+\eta^{2} \beta^{2}}$ we have

$$
\left\|y^{k+1}-y^{*}\right\| \leq\left(1-a^{k}\right)\left\|y^{k}-y^{*}\right\|+a^{k} \sigma\left\|y^{k}-y^{*}\right\|+a^{k} \eta \tau\left\|x^{k}-x^{*}\right\| .
$$


It follows from (2.3) and (2.4) that

$$
\begin{aligned}
\| x^{k+1}- & x^{*}\|+\| y^{k+1}-y^{*} \| \\
\leq & \left(1-a^{k}\right)\left\|x^{k}-x^{*}\right\|+a^{k} \theta\left\|x^{k}-x^{*}\right\|+a^{k} \eta \tau\left\|x^{k}-x^{*}\right\| \\
& +\left(1-a^{k}\right)\left\|y^{k}-y^{*}\right\|+a^{k} \sigma\left\|y^{k}-y^{*}\right\|+a^{k} \rho v\left\|y^{k}-y^{*}\right\| \\
= & {\left[1-(1-\delta) a^{k}\right]\left(\left\|x^{k}-x^{*}\right\|+\left\|y^{k}-y^{*}\right\|\right) } \\
\leq & \prod_{j=0}^{k}\left[1-(1-\delta) a^{j}\right]\left(\left\|x^{0}-x^{*}\right\|+\left\|y^{0}-y^{*}\right\|\right),
\end{aligned}
$$

where $\delta=\max \{\theta+\eta \tau, \sigma+\rho \nu\}$ and $H_{1} \times H_{2}$ is a Banach space under the norm $\|\cdot\|^{*}$.

Since $\delta<1$ and $\sum_{k=0}^{\infty} a^{k}$ is divergent, it follows that

$$
\lim _{k \rightarrow \infty} \prod_{j=0}^{k}\left[1-(1-\delta) a^{j}\right]=0 \quad \text { as } k \rightarrow \infty \text {. }
$$

Therefore

$$
\left\|x^{k+1}-x^{*}\right\|+\left\|y^{k+1}-y^{*}\right\| \rightarrow 0
$$

and consequently the sequence $\left\{\left(x^{k}, y^{k}\right)\right\}$ converges strongly to $\left(x^{*}, y^{*}\right)$, a solution to the SNVI (1.1)-(1.2) problem. This concludes the proof.

\section{References}

[1] S. S. Chang, Y. J. Cho and J. K. Kim, "On the two-step projection methods and applications to variational inequalities", Math. Inequal. Appl., accepted.

[2] L. S. Liu, "Ishikawa and Mann iterative process with errors for nonlinear strongly accretive mappings in Banach spaces", J. Math. Anal. Appl. 194 (1995) 114-127.

[3] Z. Liu, J. S. Ume and S. M. Kang, "Generalized nonlinear variational-like inequalities in reflexive Banach spaces", J. Optim. Theory Appl. 126 (2005) 157-174.

[4] H. Nie, Z. Liu, K. H. Kim and S. M. Kang, "A system of nonlinear variational inequalities involving strongly monotone and pseudocontractive mappings", Adv. Nonlinear Var. Inequal. 6 (2003) 91-99.

[5] R. U. Verma, "Nonlinear variational and constrained hemivariational inequalities", ZAMM: Z. Angew. Math. Mech. 77 (1997) 387-391.

[6] R. U. Verma, "Projection methods, algorithms and a new system of nonlinear variational inequalities", Comput. Math. Appl. 41 (2001) 1025-1031.

[7] R. U. Verma, "Generalized convergence analysis for two-step projection methods and applications to variational problems", Appl. Math. Lett. 18 (2005) 1286-1292.

[8] R. Wittmann, "Approximation of fixed points of nonexpansive mappings", Arch. Math. (Basel) 58 (1992) 486-491.

[9] E. Zeidler, Nonlinear Functional Analysis and its Applications II/B (Springer-Verlag, New York, 1990). 